\title{
Numerical Analysis of Detailed Flow Structures of a Bubble Plume*
}

\author{
Yuichi MURAI** and Yoichiro MATSUMOTO***
}

\begin{abstract}
Detailed flow structure of bubbly flows observed in a bubble plume is investigated numerically using the Euler-Lagrange model where the bubbly flow is treated as a continuum and each bubble is tracked in the flow. The governing equations are formulated with emphasis on the translational motion of the bubble in non-uniform unsteady liquid flow. Improvement of the present numerical method is confirmed by comparison with the Eulerian method, and the numerical results are validated by the comparison with the two kinds of experimental results, i.e., global flow pattern visualized by direct-lighting method, and local bubble motion measured by particle imaging velocimetry. In this paper, two-dimensional bubble plume which is confined by two parallel plates is analyzed and the parametric dependency, such as influence of the bubble size distribution and void fraction, on the whole two-phase behavior is clarified and experimental validation is conducted.
\end{abstract}

Key Words: Bubbly Flow, Multiphase Flow, Finite Differential Method, Computational Fluid Dynamics, Lagrangian Tracking Method, Natural Convection

\section{Introduction}

Bubbly two-phase flows have various flow structures which are not observed in single-phase flow, due to the complexity of translational motion and volumetric change of the bubble. The variety of the flow structure often considerably affects the performance of hydraulic machinery and chemical and bio reactors in which the bubbly media is used as a working fluid. Especially, the local flow behavior such as mutual interaction between bubble and vortex, has influence to the statistical characteristics of the flow. For instance, 1 ) generation and deformation of vortex in liquid phase due to the inhomogeneous buoyancy caused by the local distribution of bubbles, 2) accumulation of bubbles in the vortex core and turbulence generation due to the bubble migration, enhance the flow instability and the turbulence modification. Many kinds of numerical methods for gas-liquid two-

* Received 27th March, 1998. Japanese original: Trans. Jpn. Soc. Mech. Eng., Vol.63, No.611, B(1997), p. 2277-2282 (Received 11th September, 1996)

** Department of Mechanical Engineering, Fukui University, 3-9-1 Bunkyo, Fukui 910-8507, Japan

*** Department of Mechanical Engineering, The University of Tokyo, 7-3-1 Hongo, Bunkyo-ku, Tokyo 1138656, Japan phase flow have been developed recently ${ }^{(1),(2)}$. TwoFluid model, which is one of the popular models, is often used for a practical analysis of two-phase flows, such as the TRAC $\operatorname{code}^{(3)}$, RELAP $\operatorname{code}^{(4)}$ and Adiabatic model ${ }^{(5)}$. However, there are few reports that describes the numerical method by which both detailed flow structure mentioned above and global flow pattern are able to be analyzed with experimental agreement. The authors reported in the previous paper $^{(6)}$ about the three-dimensional structure of a bubble plume using the Eulerian method in which bubble motion is simulated by solving the transport equation of bubble number density. The bubble plume is a suitable object to analyze the detailed flow structure in bubbly flow because it involves various interaction between the bubble and the ambient liquid flow when the bubble rises from the bottom to the upper free surface.

In the present research, the numerical method for bubbly flow using the Lagrangian method, by which the position, velocity and volume of individual bubble are directly calculated, is established. Also the present numerical results are compared to those of the Eulerian method, and verified by comparison with experimental results obtained by flow visualization and velocity measurement using the particle imaging velocimetry. In this paper, the two-dimensional 
behavior is analyzed because the flow structure can be easily discussed compared to that of three-dimensional behavior.

\section{Governing Equations}

Governing equations for bubbly flow are described by Eulerian equation for continuous phase and Lagrangian equation for dispersed phase respectively. The Lagrangian description for bubble has higher spatial resolution than the volume-averaged model like the Two-Fluid Model. The following assumptions are employed to construct the set of equations.

(1) Bubble size is smaller than the characteristic length of the flow. Local bubble motion in a controlvolume is described by the volumetric and translational motion equations.

(2) Liquid phase is incompressible. Compressibility of bubbly flow is due to the volumetric change of bubbles.

(3) Coalescence and fragmentation of the bubbles are neglected since the void fraction treated in the present analysis is less than 0.1 .

(4) Gas inside the bubble is non-condensable and obeys the perfect gas law. Also there is no mass transfer at the bubble interface. Thus, the amount of gas inside the bubble is constant.

- Gas Volume Fraction

The local gas volume fraction $f_{G}$ is approximately given by :

$$
f_{G}=\sum_{k=1}^{N} \frac{V_{G k}}{V}=\sum_{k=1}^{N} \frac{4 \pi r^{3} G k}{3 V} \quad\left(V_{G k} \ll V\right), f_{L}=1-f_{G},
$$

where, $V$ is the averaging volume, $N$ is the number of bubbles in the volume, $r_{G k}$ is the $k$ th bubble radius, and $f_{L}$ is liquid volume fraction.

- Equilibrium Equation of Bubble Volume

The bubble volume changes due to the ambient pressure, and it is given in dynamical and thermal equilibrium condition by ;

$$
p_{G 0} V_{G 0 k}=\left(p_{L}-\frac{1}{4} \rho_{L}\left|\boldsymbol{u}_{S k}\right|^{2}\right) V_{G k}, \quad \boldsymbol{u}_{S k}=\boldsymbol{u}_{G k}-\boldsymbol{u}_{L},
$$

where, suffix 0 indicates standard condition of gas. $p_{G}$ and $V_{G}$ are the pressure and the volume of the bubble. The bubble is assumed to be in equilibrium with the surrounding pressure because the characteristic time is much smaller than that of the bubble plume and it does not affect the whole flow structure.

- Conservation Equation of Liquid Mass

$$
\frac{\partial f_{L} \rho_{L}}{\partial t}+\nabla \cdot f_{L} \rho_{L} \boldsymbol{u}_{L}=0, \rightarrow \frac{\partial f_{L}}{\partial t}+\nabla \cdot f_{L} \boldsymbol{u}_{L}=0,
$$

where $\rho_{L}$ is mass density of liquid and constant. Consequently, this equation shows the conservation of liquid volume fraction.

$$
\begin{aligned}
& \text { Conservation Equation of Total Momentum } \\
& \begin{array}{l}
\frac{\partial f_{L} \rho_{L} \boldsymbol{u}_{L}}{\partial t}+\nabla \cdot\left(f_{L} \rho_{L} \boldsymbol{u}_{L} \boldsymbol{u}_{L}\right)+\frac{\partial f_{G} \rho_{G} \boldsymbol{u}_{G}}{\partial t} \\
\quad+\nabla \cdot\left(f_{G} \rho_{G} \boldsymbol{u}_{G} \boldsymbol{u}_{G}\right) \\
\quad=-\nabla p-\left(f_{L} \rho_{L}+f_{G} \rho_{G}\right) \boldsymbol{g}+\nabla \cdot\left(1+f_{G}\right) \mu_{L}\left\{\nabla \boldsymbol{u}_{L}\right. \\
\left.\quad+\left(\nabla \boldsymbol{u}_{L}\right)^{r}-\frac{2}{3}\left(\nabla \cdot \boldsymbol{u}_{L}\right) \boldsymbol{I}\right\},
\end{array}
\end{aligned}
$$

where $\mu_{L}$ is viscosity of liquid and $\boldsymbol{g}$ is the gravitational acceleration. This equation is derived by coupling two conservation equations of momentum for two phases. The viscous term is modeled by using continuous phase component of velocity and effective viscosity due to the presence of dispersed bubbles.

- Translational Motion Equation of Bubble

Various force acts on a bubble when the bubble moves through liquid flow which has non-uniformity and unsteadiness. The force consists of following components, i.e., self inertial force, added inertial force, pressure force, viscous force, drag force, lift force, gravity force, and historical force. Here, the historical force like the Basset force for a spherical bubble is ignored because there is little vorticity generation on the bubble surface ${ }^{(7)}$.

$$
\begin{aligned}
& \frac{d}{d t}\left\{\left(\rho_{G}+\beta \rho_{L}\right) V_{G k} \boldsymbol{u}_{G k}\right\}-\frac{D_{L}}{D t}\left(\beta \rho_{L} V_{G k} \boldsymbol{u}_{L}\right) \\
& \quad=V_{G k}\left\{-\nabla p+\mu_{L}\left\{\nabla^{2} \boldsymbol{u}_{L}+\frac{1}{3} \nabla\left(\nabla \cdot \boldsymbol{u}_{L}\right)\right\}\right\} \\
& \quad-\frac{1}{2} \rho_{L} \pi r_{G k}{ }^{2} C_{D}\left|\boldsymbol{u}_{S k}\right| \boldsymbol{u}_{S k}-C_{L} \rho_{L} V_{G k}\left\{\boldsymbol{u}_{S k} \times\left(\nabla \times \boldsymbol{u}_{L}\right)\right\} \\
& \quad-\rho_{G} V_{G k} \boldsymbol{g},
\end{aligned}
$$

where $\beta$ is added mass coefficient for a spherical bubble, $C_{D}$ is drag coefficient, $C_{L}$ is lift coefficient, and these are given by

$$
\begin{aligned}
& C_{D}=\max \left\{\frac{48}{\operatorname{Re}}\left(1-\frac{2.21}{\sqrt{R e}}\right), \frac{16}{\operatorname{Re}}\right\}, \\
& \operatorname{Re}<200, R e=\frac{2 r_{G} \rho_{L}\left|\boldsymbol{u}_{S k}\right|}{\mu_{L}}, \\
& \frac{D_{L}}{D t}=\frac{\partial}{\partial t}+\boldsymbol{u}_{L} \cdot \nabla, C_{L}=\frac{1}{2}, \beta=\frac{1}{2}, f_{G}<0.10 .
\end{aligned}
$$

Here the drag coefficient of bubble is given by two theoretical formulas of Stokes law and Moore ${ }^{(8)}$, and the lift coefficient is given by Auton ${ }^{(9)}$, which are applicable to a small spherical gas bubble in wellpurified water or clean oil.

- Tracking Equation of Bubble Position

$$
\boldsymbol{X}_{G k}(t)=\boldsymbol{X}_{G 0 k}+\int_{0}^{t} \boldsymbol{u}_{G k}(t) d t,
$$

here $\boldsymbol{X}_{G 0}$ is the initial position vector of the bubble.

\section{Numerical Method}

The governing equations are solved by a semiimplicit method based on the HSMAC algorithm which was originally developed by Hirt \& $\mathrm{Cook}^{(10)}$ for incompressible viscous single phase flow. The HSMAC method is also available for the calculation 
of bubbly two-phase flow which has both bubbly (compressible) region and non-bubbly (incompressible) region if an adequate under-relaxation factor is adapted in the iterating process to satisfy continuity condition of two volume fractions; $f_{G}+f_{L}=1$. Also, it is simplified to develop the computation procedure by using the HSMAC algorithm for the present governing equations which consist of Eulerian part and Lagrangian part. This is because solving the Poisson equation of pressure is not required in the HSMAC method. Thus, the present numerical procedure is as follows.

(1) Calculate new translational velocity of bubble by Eqs. (5), (6) and ( 7 ).

(2) Calculate locations of bubbles by using the translational velocity by Eq. ( 8 ).

(3) Calculate new liquid velocity by Eq. (4).

(4) Calculate new bubble volume by Eq. (2).

(5) Calculate new gas volume fraction by Eq. (1).

(6) Calculate new liquid volume fraction by Eq. (3).

(7) Calculate volume fraction error and pressure correction value which is described by Eq. ( 9 ).

(8) Go to the next time step if the volume fraction error is converged to zero.

The pressure correction value used in process $(7)$ is given by the next equation which is derived by the principle of the HSMAC method.

- Pressure Correction Equation

$$
\delta p=-\varepsilon /\left\{\frac{2 \delta t^{2}}{\rho_{L}}\left(\frac{1}{\delta x^{2}}+\frac{1}{\delta y^{2}}+\frac{1}{\delta z^{2}}\right)+\frac{f_{G}}{p}\right\},
$$

where $\varepsilon=f_{L}+f_{G}-1$ is the error of continuity condition of volume fractions, $\delta t$ and $\delta x$ are the time step and the spatial interval of discretized governing equations respectively.

\section{Comparison of Lagrangian Method with Eulerian Method}

The Lagrangian method has basically higher spatial resolution than the Eulerian method since the bubble motion is represented by equations which are not spatially averaged. In this chapter, the comparison of two numerical results for a bubble plume is shown to confirm the improvement of spatial resolution.

Figure 1 shows the comparison of instantaneous distribution of the bubble and stream function of liquid phase in a two-dimensional bubble plume which condition is shown in Table 1. In order to avoid the influence of numerical diffusion in the Eulerian method, the CIP scheme ${ }^{(11)}$ is adapted as spatial differencing in conservation equation of bubble number density ${ }^{(6)}$, because incontinuous profile can be tracked with enough precision by the CIP scheme. As shown in the figure, both results, such as periodic oscillation of the bubble plume, have good agreement in the case of large bubble injection. However, the convergence and the meandering instability of the

Table 1 Calculation condition for comparison

\begin{tabular}{|l|l|}
\hline Liquid $\rho_{L}=10^{3} \mathrm{~kg} / \mathrm{m}^{3}$ & Tank Width $L=0.300 \mathrm{~m}$ \\
\hline Gas $\rho_{0}=1.25 \mathrm{~kg} / \mathrm{m}^{3}$ & Height $\quad \mathrm{H}=0.300 \mathrm{~m}$ \\
\hline Kinematic Viscosity $\nu_{L}=5 \times 10^{-0} \mathrm{~m}^{2} / \mathrm{s}$ \\
\hline Bubble Injection Width $B=0.060 \mathrm{~m}$ (at center) \\
\hline Void Fraction at Injector $\mathrm{f}_{\mathrm{O}}=0.05$ (Average) \\
\hline
\end{tabular}
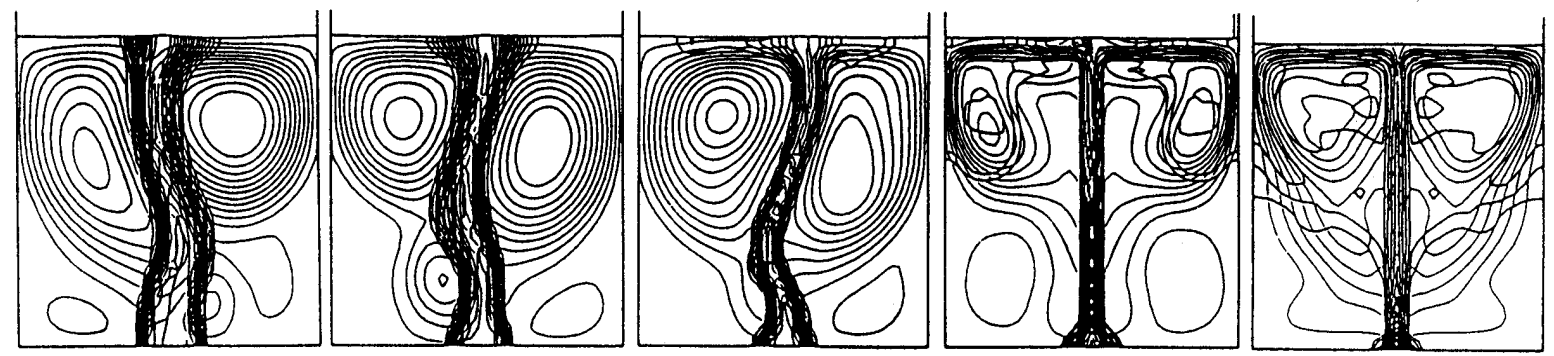

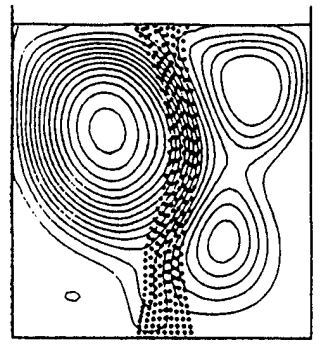

$\mathrm{R}=1.6 \mathrm{~mm}$

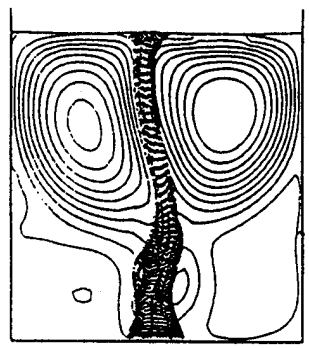

$\mathrm{R}=0.8 \mathrm{~mm}$

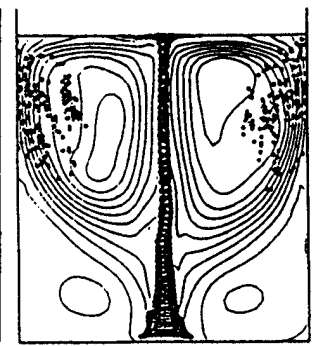

$\mathrm{R}=0.4 \mathrm{~mm}$

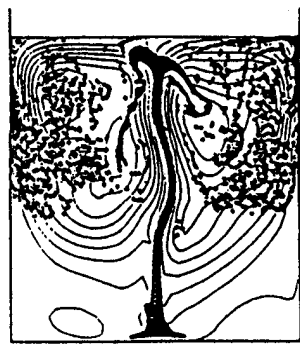

$\mathrm{R}=0.2 \mathrm{~mm}$

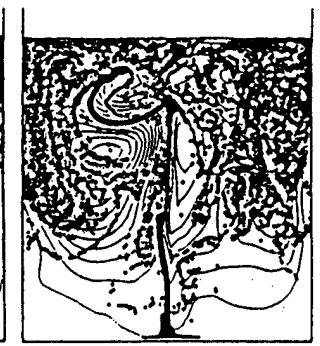

$\mathrm{R}=0.1 \mathrm{~mm}$

Fig. 1 Comparison of Eulerain and Lagrangian methods (Top: Eulerian method with CIP scheme, Bottom: Lagrangian method) 
bubble plume due to the local interaction between bubble and liquid flow in the case of small bubble are not well simulated by the Eulerian method. This indicates that spatial resolution of the Eulerian method is worse compared to the Lagrangian method even though higher order spatial differencing scheme is used. In other words, the Lagrangian method has advantage in analyzing detailed flow structure which includes strong local gas-liquid interaction. On the other hand, the demerit of the Lagrangian method is the increase of computational load in case of large scale bubbly flow which has a large number of bubbles.

\section{Influence of Bubble Size Deviation}

Generally, a bubbly flow has large deviation in bubble size. In this case, the Eulerian method should be modified to the method in which dispersed phase is divided into several groups according to the size differences. To the contrary, it can be treated directly in the Lagrangian method.

Figure 2 shows the influence of bubble size deviation on the bubble plume structure, where flow condi-

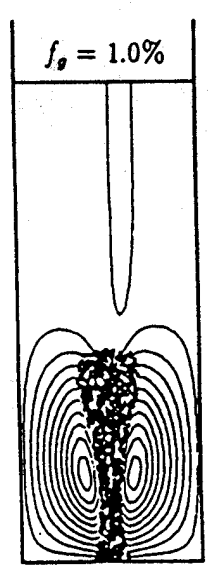

$\leftarrow$

$R v=0.01 \mathrm{~mm}$

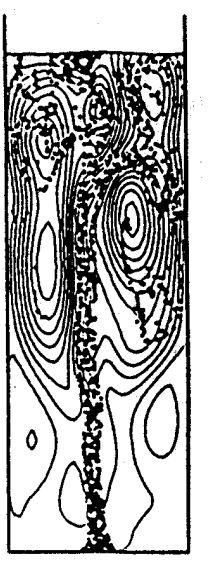

$\leftarrow$

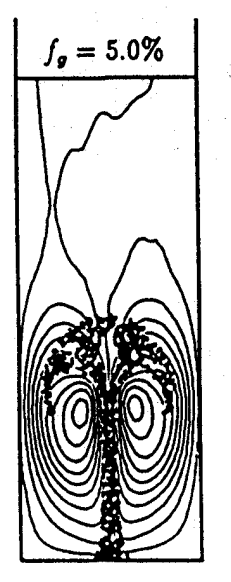

$R v=0.01 \mathrm{~mm}$

$R v=0.20 \mathrm{~mm}$

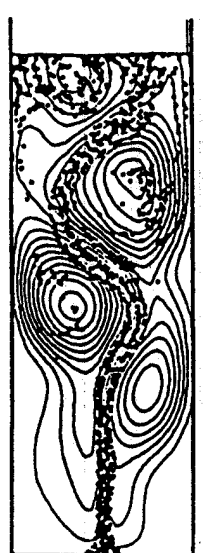

$R m=1.0 \mathrm{~mm}$

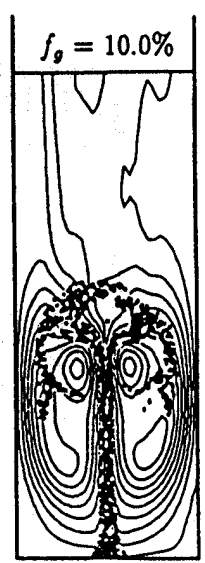

$R v=0.50 \mathrm{~mm}$
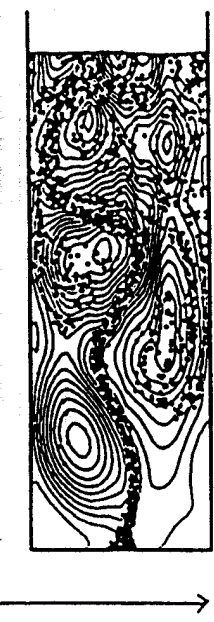

tion is the same as shown in Table 1 but free surface level is $H=0.60 \mathrm{~m}$. Here, $R_{m}$ is averaged bubble radius (number-averaged value, mm), and $R_{v}$ is standard deviation of bubble radius $(\mathrm{mm})$. As shown in top-figure, the bubble number density distributes incontinuously at the top region of the bubble plume in case of small $R_{v}$ just after starting bubble injection. To the contrary, it becomes diffusely in case of large $R_{v}$ since bubble rising velocity includes large deviation.

Numerical predicted results obtained by the Eulerian method sometimes show very similar profile to experimental results even though uniform bubble size is assumed in computation. However it must be recognized that the effect of numerical diffusion is essentially different to that of bubble size deviation. The small fluctuation of stream line in upper region of the tank is caused by liquid-lifting due to intermittent injection of the individual bubble.

Bottom figures represent the influence of $R_{v}$ in well-developed flow. Many bubbles stay under the free surface in case of large $R_{m}$ because the injection ratio of small bubbles is higher for the large $R_{v}$. To

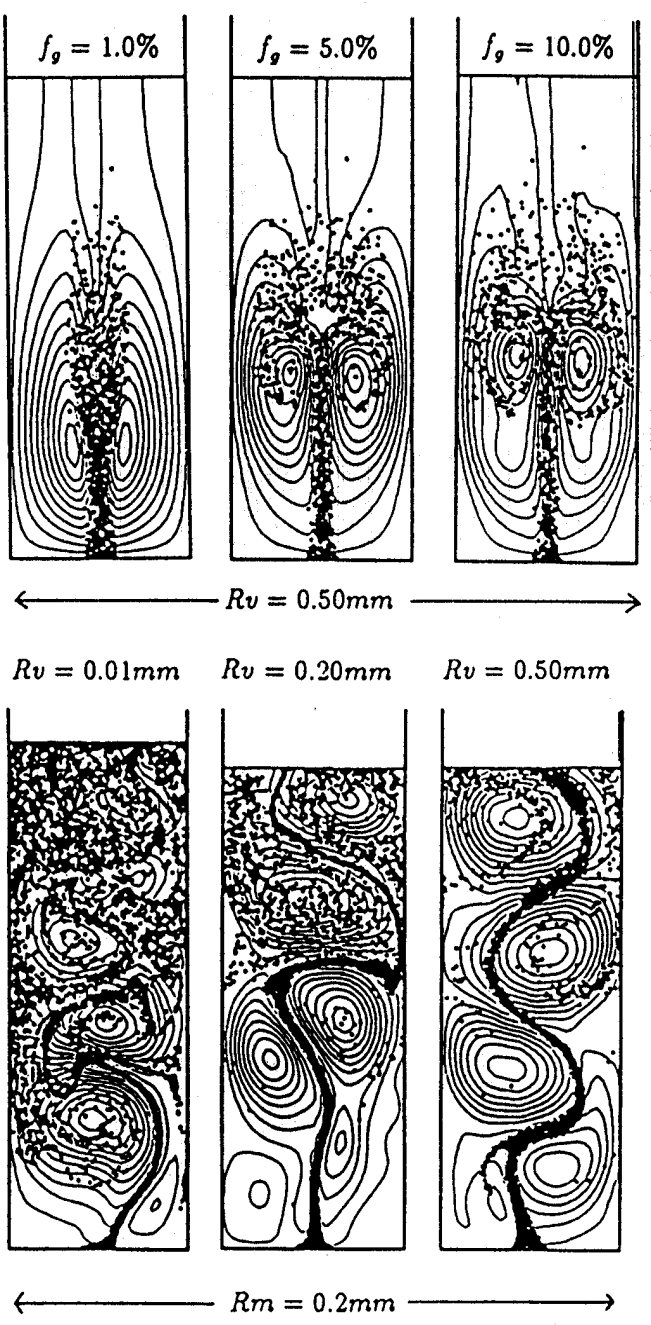

Fig. 2 Effect of deviation of bubble size (Top: starting structure, Bottom: developed structure) 
the contrary, almost all bubbles are released to outside in case of large $R_{v}$ since small bubbles are carried by vertical liquid flow which is strongly driven by large bubbles.

\section{Comparison with Experiment and Discussion}

Two kinds of experiments are conducted to verify the present numerical method by two-dimensional bubble plume in a thin tank. One is visualization of airwater bubble plume (Exp. A), and the other is PIV (Particle Imaging Velocimetry) measurement of $\mathrm{N}_{2}{ }^{-}$ Silicon oil bubble plume. The experimental condition is shown in Table 2. The authors' preveous papers $^{(12),(13)}$ are recommended for referencing the detailed experimental method.

\section{1 Exp. A : Validation by visualization}

It is confirmed by experimental observation that the confined liquid flow inside the two parallel plates is
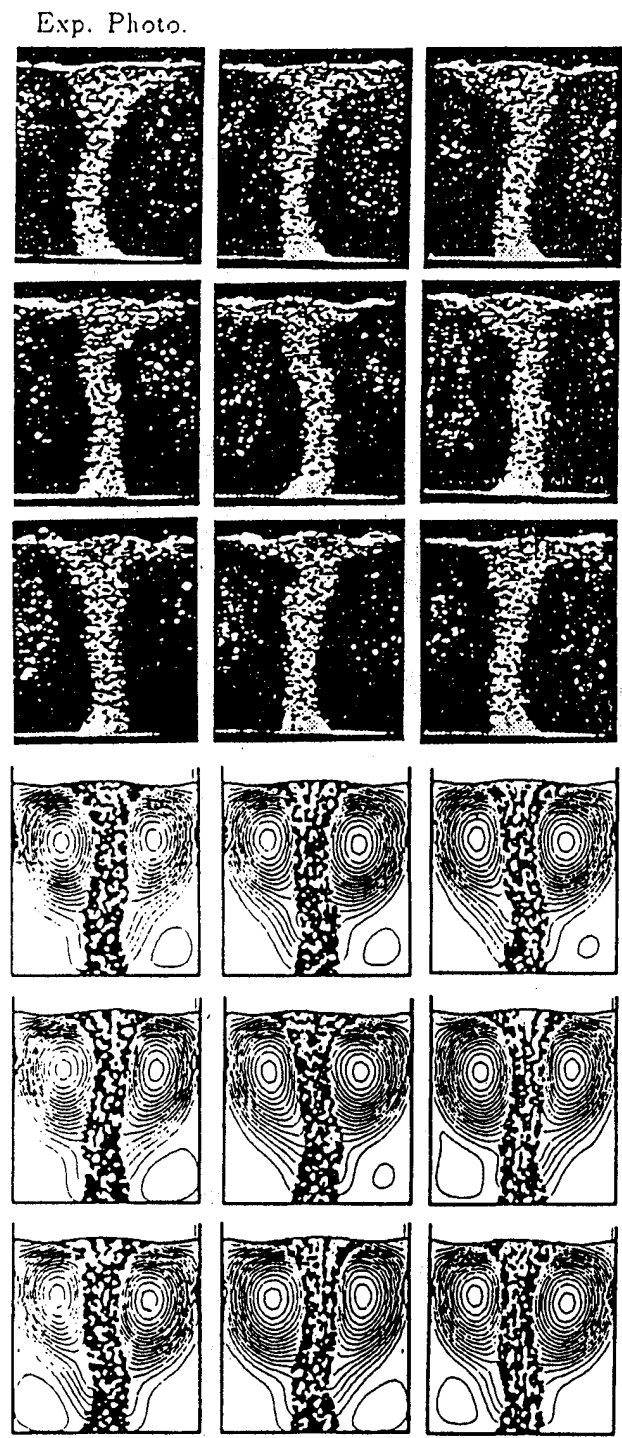

Stream Line

Table 2 Experimental condition

\begin{tabular}{|c|c|}
\hline \multicolumn{2}{|c|}{ Experimental Condition (Common) } \\
\hline Tank Size $L=0.300 \mathrm{~m}$ & $H \max =0.9 \mathrm{~m}$ \\
\hline Tank Depth $W=0.020 \mathrm{~m}$ & In jection $B=0.080 \mathrm{~m}$ \\
\hline Nozzle Number $=17 \times 3$ & Nozzle dia. $=0.19 \mathrm{~mm}$ \\
\hline Atm. Prs. $P a=101.3 \mathrm{kPa}$ & $18 \sim 21^{\circ} \mathrm{C}$ \\
\hline Experiment $(A)$ & Experiment (B) \\
\hline $\begin{array}{lc}\text { Liquid } & 1000 \mathrm{~kg} / \mathrm{m}^{3} \\
\text { Gas } & 1.25 \mathrm{~kg} / \mathrm{m}^{\mathrm{s}} \\
\text { K.Visc. } & 1 \times 10^{-0} \mathrm{~m}^{2} / \mathrm{s}\end{array}$ & $\begin{array}{lr}\text { Liquid } & 960 \mathrm{~kg} / \mathrm{m}^{3} \\
\text { Gas } & 1.22 \mathrm{~kg} / \mathrm{m}^{3} \\
\text { K.Visc. } & 5 \times 10^{-6} \mathrm{~m}^{2} / \mathrm{s}\end{array}$ \\
\hline $\begin{array}{ll}\text { Mean Rad. } & R m=1.10 \mathrm{~mm} \\
\text { Deviation } & R v=0.20 \mathrm{~mm} \\
\text { Void Frac. } & f_{0}=0.080\end{array}$ & $\begin{array}{l}\text { Two Injection Cond. } \\
R m_{1} R v_{1} f_{0} \text { are } \\
\text { indicated in Figs. }\end{array}$ \\
\hline $\mathrm{H}=0.300 \mathrm{~m}$ & $\mathrm{H}=0.600 \mathrm{~m}$ \\
\hline & Zivertical \\
\hline $\begin{array}{l}\text { Tuft Length } 20 \mathrm{~m} \\
\text { Tuft Interval } 20 \mathrm{~mm}\end{array}$ & M) $Z=0.250 \sim 0.350 \mathrm{~m}$ \\
\hline
\end{tabular}
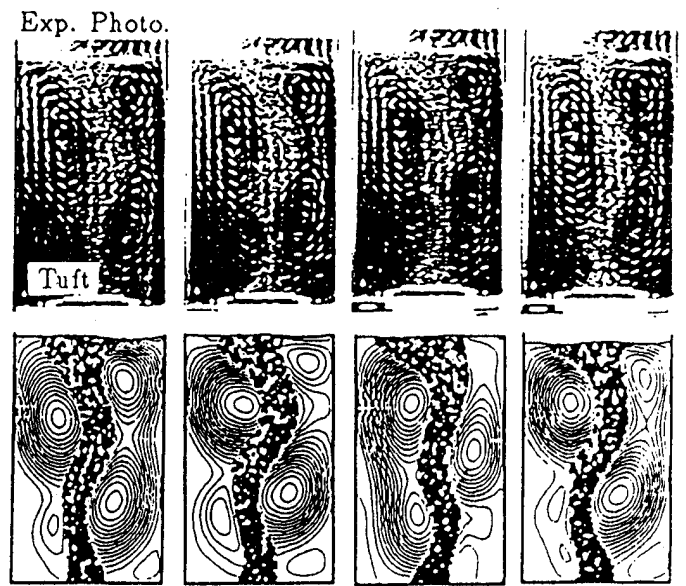

Num. Result

(b) $H=0.45 \mathrm{~m}$, meandering behavior, time interval: $5 \mathrm{sec}$
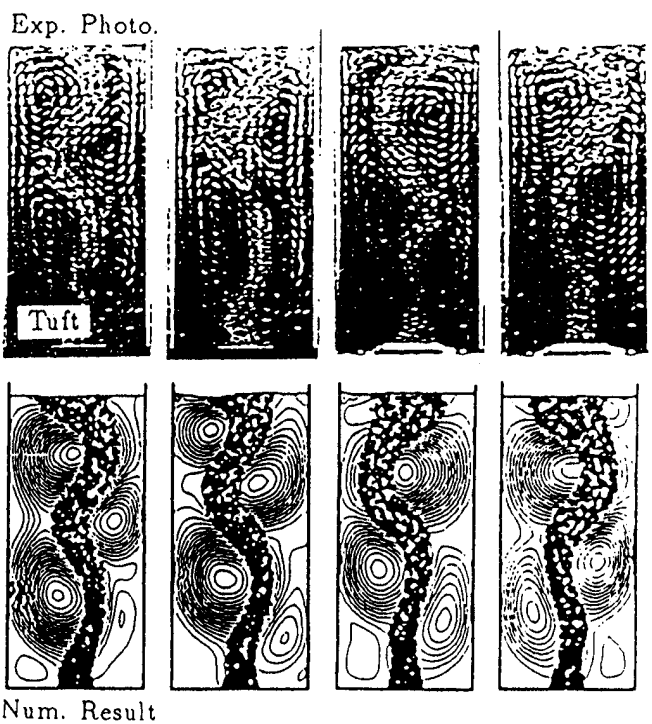

(a) $H=0.30 \mathrm{~m}$, swaying behavior, time interval: $5 \mathrm{sec}$

(c) $H=0.60 \mathrm{~m}$, meandering behavior, time interval: $5 \mathrm{sec}$

Fig. 3 Comparison of computational and experimental results 
turbulent flow because the Reynolds number as HeleShaw flow is over 4000 . The turbulent flow behavior can not be treated directly by the present Lagrangian model equations because the model is basically formulated by spatially-averaged variables and no turbulence model is included to describe the liquid flow. Therefore, two-dimensional calculation, in which wall turbulent friction is considered as source term in momentum conservation equation, is applied.

Comparison between numerical and experimental results on Exp. A is shown in Fig. 3. The small segments in the experimental photograph and curves in predicted results represent visualizing-tufts and stream lines respectively. The following matters are made clear from the figure. (1) The bubble plume shows the stronger unsteady meandering behavior ${ }^{(14)}$ in case of the higher free surface. (2) The meandering behavior is caused by the interaction between rising bubbles and liquid circulation in the tank. ( 3 ) Numerical and experimental results are in good agreement on above two matters.

Here, time dependency of the tuft against fluctuation of liquid flow and approximation of the wall turbulent friction in two-phase media are expected to have some problems in validity. However, it can be confirmed that the Lagrangian method has certain performance to predict the macroscopic behavior of the bubble plume even though it is in turbulent flow condition. Nearly similar results of such macroscopic behavior can be obtained by Two-Fluid model by taking care of validity of constitutive equations ${ }^{(5)}$. From here, experimental validation on the internal flow structure of the bubble plume, which is expected not to be simulated sufficiently by the Eulerian method or Two-Fluid model, is discussed.

\section{2 Exp. B : Validation by PIV measurement}

Figure 4 represents comparison between predicted results and PIV measured results of rising velocity profile of the bubble $\left(V_{G}\right)$ at three horizontal crosssections in the bubble plume. In the figure, $\mathrm{X}$ is horizontal coordinate, $\mathrm{L}, \mathrm{M}$ and $\mathrm{H}$ indicates the measuring location which corresponds to the vertical coordinates shown in Table 2. Three-dimensional calculation is conducted by using $30 \times 80 \times 10$ grid space, and the bubble motion is measured by twodimensional PIV in area of $150 \mathrm{~mm} \times 100 \mathrm{~mm}$. Both results are obtained by statistical sampling for 10.0 seconds in well-developed-flow condition in which the whole behavior of the bubble plume has laminar-like, and almost axisymmetric structure compared to Exp. A. From the figure, the followings are clarified:

(1) Rising velocity profile of the bubble is parabolic at location L, and has little scattering due to the deviation of the bubble size. (2) Curvature of the profile becomes sharp in case of high void fraction. (3) The profile at location $\mathrm{M}$ shows two combined structure which consists of parabolic and uniform profiles. (4) These three terms are in good agreement between predicted and measured results. ( 5 ) There is clear discrepancy in the profile measured at location
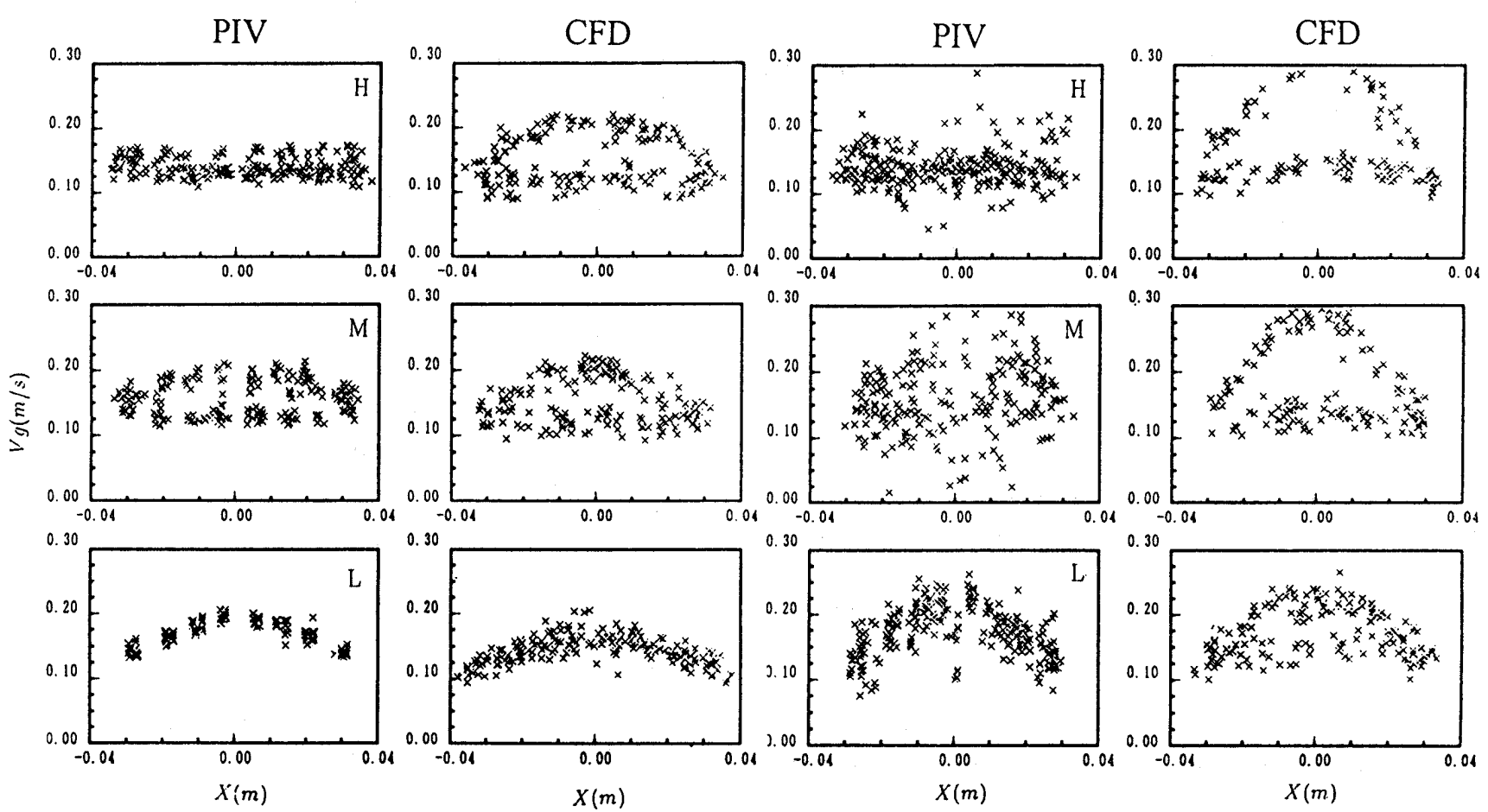

(a) $f_{c}=0.53 \%, R_{m}=0.67 \mathrm{~mm}$

(b) $f_{c}=1.97 \%, R_{m}=0.71 \mathrm{~mm}$

Fig. 4 Comparison of computational and PIV results for bubble rising velocity 
H.

Here, terms (1) and (2) are caused by the nearly Gaussian profile of the rising velocity profile of the liquid which is induced by the bubble buoyancy. Two kinds of the profile in term (3) appears due to existence of two kinds of bubble behavior, i.e., sliding bubbles on the wall surface and straightly rising bubbles in the center of the channel. Reasons of the discrepancy in term ( 5 ) is thought to be caused by the following factors ; uncertainty in magnitude of lift force acting on the bubble in strong shear flow field, lack of spatial resolution in computation using averaged equations, difficulty to give strict boundary condition for bubble motion near the wall.

On the lift force of bubble, as reported by Auton ${ }^{(9)}$, Saffman ${ }^{(15)}$ and Sridhar \& Kat $z^{(16)}$, the standard description of the lift force of bubble has not been clarified exactly. In the present research, while Auton's lift force is used, we can estimate from the experimental results that the actual lift force is stronger than Auton's lift force. In addition, bubble-bubble interactive force which is produced by the wake effect or neighboring flow of the bubble, is also thought to be one of the factor which gives deviation in rising velocity of the bubble. The present PIV results and numerically predicted results do not have good correspondence especially at location $\mathrm{H}$ due to several unknown factors discussed above. However, except for the uncertainties in lift force and bubble-bubble interactive force, the numerical and experimental two-dimensional flow structures of bubble plume have qualitatively good agreement.

Thus, not only the global behavior which is shown in section 5.1, but also the bubble motion inside the bubble plume, are well simulated by the present numerical analysis using the Lagrangian method. In the future, the generation mechanism and quantitative study for lift force and bubble-bubble interactive force should be elucidated and introduced into the present governing equations.

\section{Conclusion}

A numerical simulation method of bubbly flow using the Lagrangian method in which position, velocity vector and volume of individual bubble are directly tracked, was established. The present method was verified by comparison with experimental results in a two-dimensional bubble plume, and followings were made clear.

(1) The numerical method of bubbly flow using the Lagrangian method has an advantage in the simu- lation of detailed flow structure such as local accumulation of bubble and generation of flow instability, compared to the Eulerian method in which the bubble motion is calculated by spatial averaged equations model.

(2) The macroscopic flow patterns simulated by the present method, such as swaying and meandering behavior, have good agreement with the experimental results in water-air two-dimensional bubble plume.

(3) By PIV measurement of bubble motion in the bubble plume consisted of Nitrogen gas bubble and silicon oil, it was also made clear that local rising velocity profile of bubbles in the bubble plume was satisfactory simulated by the present numerical method.

\section{References}

(1) Matsumoto, Y. and Prosperetti, A. Edit., Proc. ISAC' 97 High Performance Computing on Multiphase Flows, (1997).

(2) The Japan Society of Nuclear Power Edit., Numerical Analysis of Gas-Liquid Two-Phase Flow (1992), p. 22-75, (in Japanese), Asakura Publisher.

(3) Liles, D. R., et al., NUREG/CR-3858, LA-10157MS, (1986)

(4) Ransom, V.H., et al., NUREG/CR-4312, EGG2398, Rev. 1., (1987).

(5) Tomiyama, A. et al., Trans. Jpn. Soc. Mech. Eng. (in Japanese), Vol. 60, No. 580, B(1994), p. 39873993.

(6) Matsumoto, Y. and Murai, Y., Trans. Jpn. Soc. Mech. Eng. (in Japanese), Vol.61, No. 588, B (1995), p. 2818-2825.

(7) Takagi, S., Doctoral Thesis of The Univ. of Tokyo,(1995), (in Japanese).

(8) Moore, D. W., J. Fluid Mech. Vol. 6 (1959), p. 113130.

(9) Auton, T. R., J. Fluid Mech., Vol. 183 (1987), p. 199-223.

(10) Hirt, C. W. and Cook, J. L., J. Comp. Phys., Vol. 10 (1972), p. 324-335.

(11) Takewaki, H. and Yabe, T., J. Comp. Physics, Vol. 70 (1987), p. 355-366.

(12) Murai, Y., Yamanishi, N. and Matsumoto, Y., Proc. 3rd Asian Symp. on Visualization, (1994), p. 398-403.

(13) Murai, Y. and Matsumoto, Y., ASME-FED, Vol. 185 (1994), p. 203-208.

(14) Alam, M. and Arakeri, V. H., J. Fluid Mech., Vol. 254 (1993), p. 363-374.

(15) Saffman, P.G., J. Fluid Mech.,Vol. 22, No. 2 (1965), p. 385-402.

(16) Sridhar, G. and Katz, J., Phys. Fluids, Vol. A 7, No. 2 (1995), p. 389-396. 